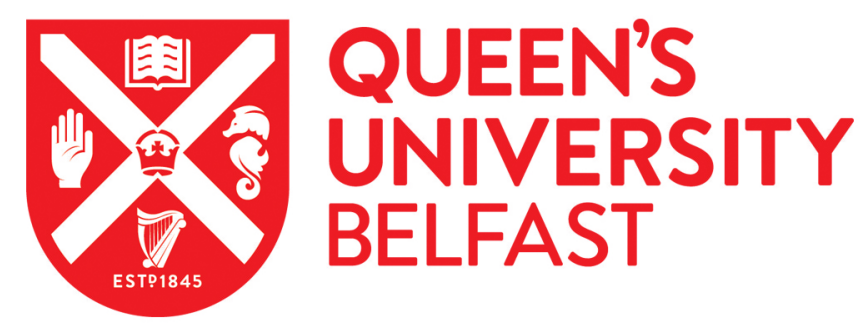

\title{
Integrated Laser-Target Interaction Experiments on the RAL Petawatt Laser
}

Patel, P. K., Key, M. H., Mackinnon, A. J., Berry, R., Borghesi, M., Chambers, D. M., Chen, H., Clarke, R. C., Damian, C., Heathcote, R., Hey, D., Eagleton, R., Freeman, R. R., Glenzer, S., Gregori, G., Izumi, N., Kar, S., King, J. A., Nikroo, A., ... Zhang, B. (2005). Integrated Laser-Target Interaction Experiments on the RAL Petawatt Laser. Plasma Physics and Controlled Fusion, 47(12 B), B833.

Published in:

Plasma Physics and Controlled Fusion

Document Version:

Publisher's PDF, also known as Version of record

Queen's University Belfast - Research Portal:

Link to publication record in Queen's University Belfast Research Portal

\footnotetext{
General rights

Copyright for the publications made accessible via the Queen's University Belfast Research Portal is retained by the author(s) and / or other copyright owners and it is a condition of accessing these publications that users recognise and abide by the legal requirements associated with these rights.
}

Take down policy

The Research Portal is Queen's institutional repository that provides access to Queen's research output. Every effort has been made to ensure that content in the Research Portal does not infringe any person's rights, or applicable UK laws. If you discover content in the Research Portal that you believe breaches copyright or violates any law, please contact openaccess@qub.ac.uk. 
Integrated laser-target interaction experiments on the RAL petawatt laser

This article has been downloaded from IOPscience. Please scroll down to see the full text article.

2005 Plasma Phys. Control. Fusion 47 B833

(http://iopscience.iop.org/0741-3335/47/12B/S65)

View the table of contents for this issue, or go to the journal homepage for more

Download details:

IP Address: 143.117.90.74

The article was downloaded on 08/09/2012 at 06:47

Please note that terms and conditions apply. 


\title{
Integrated laser-target interaction experiments on the RAL petawatt laser
}

\author{
P K Patel ${ }^{1}$, M H Key ${ }^{1}$, A J Mackinnon ${ }^{1}$, R Berry ${ }^{1}$, M Borghesi ${ }^{2}$, \\ D M Chambers ${ }^{3}$, H Chen ${ }^{1}$, R Clarke ${ }^{4}, C_{\text {Damian }}{ }^{1}$, R Eagleton ${ }^{3}$, \\ R Freeman ${ }^{5}$, S Glenzer ${ }^{1}$, G Gregori $^{1}$, R Heathcote ${ }^{4}$, D Hey ${ }^{6}$, N Izumi ${ }^{1}$, \\ S Kar ${ }^{2}$, J King ${ }^{6}$, A Nikroo ${ }^{7}$, A Niles ${ }^{1}$, H-S Park ${ }^{1}$, J Pasley ${ }^{8}$, N Patel ${ }^{5}$, \\ R Shepherd $^{1}$, R A Snavely ${ }^{1}$, D Steinman ${ }^{7}$, C Stoeckl $^{9}$, M Storm $^{9}$,

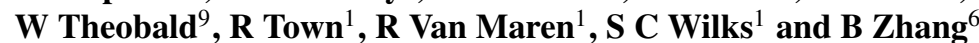 \\ ${ }^{1}$ Lawrence Livermore National Laboratory, Livermore, CA 94550, USA \\ 2 Department of Physics, Queen's University of Belfast, Belfast, BY7 1NN, UK \\ 3 Atomic Weapons Establishment, Aldermaston, Reading, RG7 4PR, UK \\ ${ }^{4}$ CCLRC Rutherford Appleton Laboratory, Chilton, Didcot, Oxon., OX11 0QX, UK \\ ${ }^{5}$ Ohio State University, Columbus, OH 43210, USA \\ ${ }^{6}$ Department of Applied Sciences, University of California Davis, Davis, CA 95616, USA \\ ${ }^{7}$ General Atomics, PO Box 85608, San Diego, CA 92186, USA \\ ${ }^{8}$ University of California, San Diego, CA 92093, USA \\ ${ }^{9}$ Laboratory for Laser Energetics, University of Rochester, Rochester, NY 14623, USA
}

Received 1 July 2005

Published 14 November 2005

Online at stacks.iop.org/PPCF/47/B833

\begin{abstract}
We review a recent experimental campaign to study the interaction physics of petawatt laser pulses incident at relativistic intensities on solid targets. The campaign was performed on the $500 \mathrm{~J}$ sub-picosecond petawatt laser at the Rutherford Appleton Laboratory. An extensive suite of optical, x-ray, and particle diagnostics was employed to characterise the processes of laser absorption, electron generation and transport, thermal and K-alpha $\mathrm{x}$-ray generation, and proton acceleration.
\end{abstract}

(Some figures in this article are in colour only in the electronic version)

\section{Introduction}

Since the development of the first high energy petawatt-class laser less than a decade ago at the Lawrence Livermore National Laboratory we have seen the rapid emergence of similar laser systems at numerous laboratories around the world [1]. This new generation of ultrashortpulse high energy lasers, able to deliver hundreds of joules of energy down a single beam line in a sub-picosecond pulse, have produced a host of remarkable discoveries and provide a valuable tool for exploring new regimes in relativistic laser-plasma physics, encompassing high energy x-rays and $\gamma$-rays, relativistic electrons, intense ion beams and superstrong magnetic fields $[2,3,4]$. The coupling in the near-future of multi-kilojoule petawatt-class lasers with 
large-scale fusion lasers - including the NIF and Omega EP (US), LIL (France) and FIREX (Japan) - will further expand opportunities in fast ignition, high energy X-ray radiography and high energy density physics research.

The $500 \mathrm{~J}$ petawatt laser at the Rutherford Appleton Laboratory is currently the highest energy short-pulse laser in the world [5]. In this paper we describe a recent experimental campaign carried out on the facility. The campaign, performed by a large collaborative team from eight different laboratories, was designed to study a variety of relativistic laserinteraction phenomena including laser absorption, fast electron transport, proton heating and high-brightness x-ray source generation. The wide scope of the experiment necessitated the deployment of a very large set of diagnostics - in total 25 separate instruments. In order to obtain the most comprehensive set of measurements we chose to field all 25 diagnostics simultaneously on every shot.

\section{Experiment}

The Vulcan Nd : glass petawatt laser delivers over $400 \mathrm{~J}$ of energy onto target in a $400 \mathrm{fs}$ FWHM (full-width at half-maximum) temporal pulse. It combines an OPCPA (optical parametric chirped-pulse amplification) front end with a mixed glass amplification chain. The amplified chirped pulse is recompressed by a pair of $94 \mathrm{~cm}$ diameter gold gratings to a $400 \mathrm{fs}$ pulsewidth before delivery to the interaction chamber, where a $65 \mathrm{~cm}$ diameter $f / 3$ off-axis parabola focuses it onto the target. In this experiment the laser was incident with p-polarization at an angle of $28^{\circ}$ to the target normal.

An extensive array of diagnostics—-some 25 instruments in total—were fielded in and around the target chamber. The front side diagnostics included a single-hit CCD for measuring $\mathrm{K}$-alpha yields, an Al K-shell x-ray spectrometer, an optical spectrometer measuring the specular reflected light, a neutron scintillator detector, an X-ray pinhole camera and an X-ray diode array. The rear side diagnostics included two XUV imagers operating at $68 \mathrm{eV}$ and $256 \mathrm{eV}$, an XUV streak camera imaging at $68 \mathrm{eV}$, an optical imaging streak camera, a multilayer hard x-ray spectrometer, a HOPG (highly oriented pyrolitic graphite) crystal x-ray spectrometer, a second single-hit K-alpha CCD camera, radiochromic film (RCF) and a megaelectron-volt electron spectrometer. The requirement for all of the diagnostics to function simultaneously on each shot introduced considerable complexity to the experimental set-up. To facilitate the design a 3D CAD model was created of the entire target chamber, as well as each instrument. This enabled a single design to be reached which optimized the locations and viewing angles of each diagnostic whilst ensuring that no interferences would exist (see figure 1).

\section{Focal spot}

The laser focal spot was measured at low energy (attenuated low energy pulses from the OPCPA) using a $\times 40$ microscope objective and 16-bit vacuum CCD directly imaging the focus of the parabola. The focal intensity distribution is an important parameter for $2 \mathrm{D}$ or 3D particle-in-cell (PIC) modelling of the laser-target interaction, particularly when one is interested in the spatial and directional characteristics of the fast electrons and their subsequent role in isochoric target heating, $\mathrm{K}$-alpha $\mathrm{x}$-ray production and proton acceleration.

Figure 2 shows an image of the laser at best focus. The focal spot has a $6.9 \mu \mathrm{m}$ FWHM. Approximately $20 \%$ of the incident laser energy is contained within this diameter. Given $400 \mathrm{~J}$ of energy on the target this yields a peak intensity of $5 \times 10^{20} \mathrm{~W} \mathrm{~cm}^{-2}$. One notes that a full $80 \%$ 


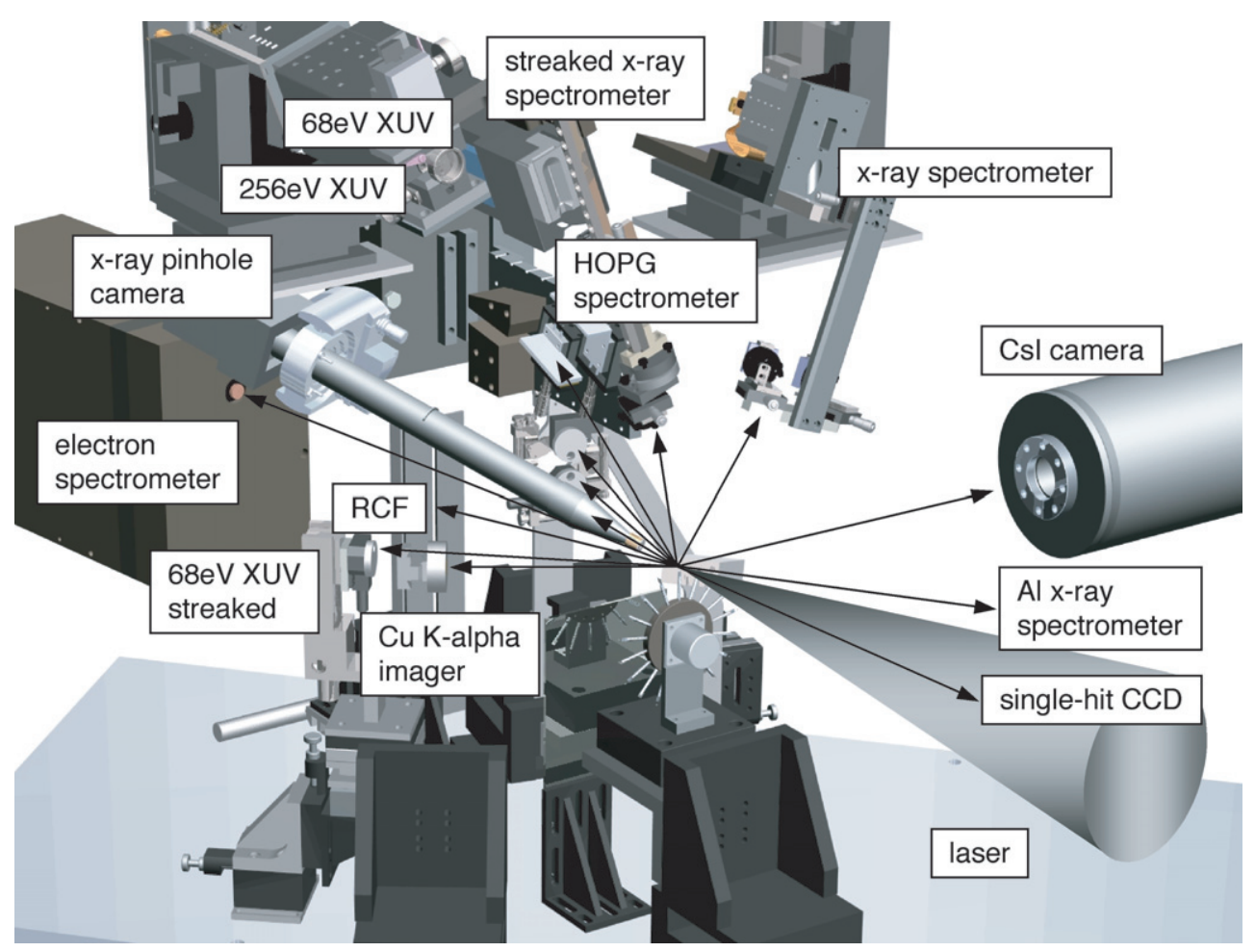

Figure 1. Schematic of experimental set-up inside the interaction chamber, highlighting a selection of the total diagnostic suite. The majority of these diagnostics are positioned to view the rear surface of the target.
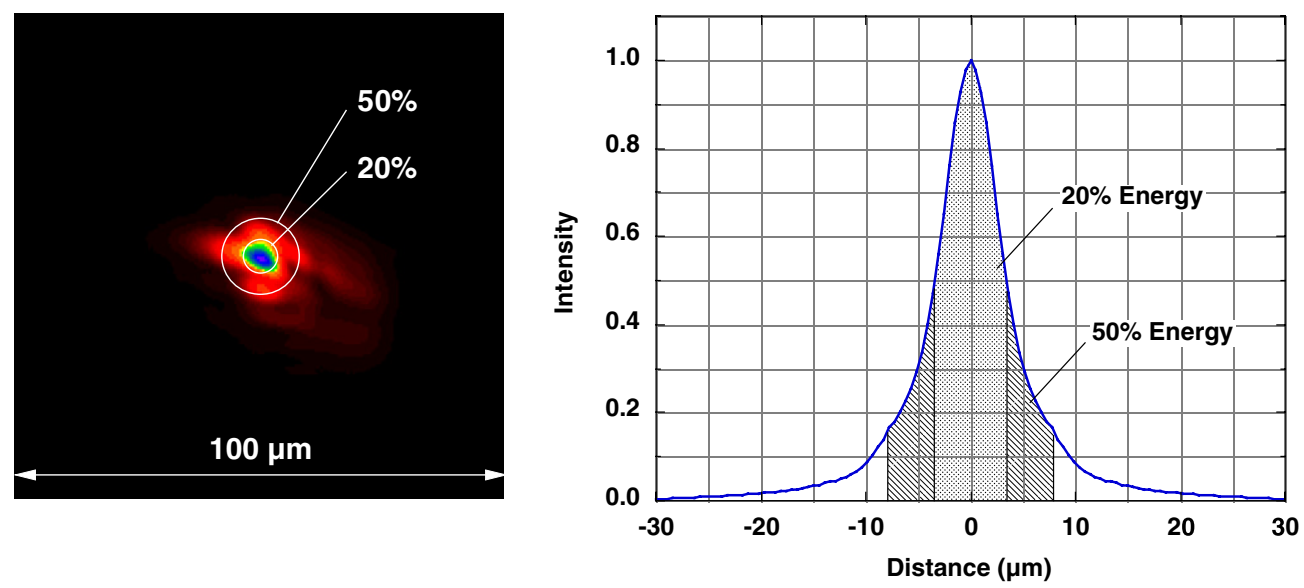

Figure 2. Image of vacuum focal spot at low energy taken with a 16-bit CCD camera (left). Radial lineout of focal spot intensity showing $20 \%$ and $50 \%$ encircled energy boundaries (right).

of the laser energy is incident on the target outside of the FWHM spot. Approximately 50\% of the total laser energy is contained within a $16 \mu \mathrm{m}$ spot diameter, with the remaining $50 \%$ extending out to a diameter of approximately $50 \mu \mathrm{m}$. Although incident at a lower intensity this energy in the wings of the distribution is still sufficiently intense so as to contribute to 

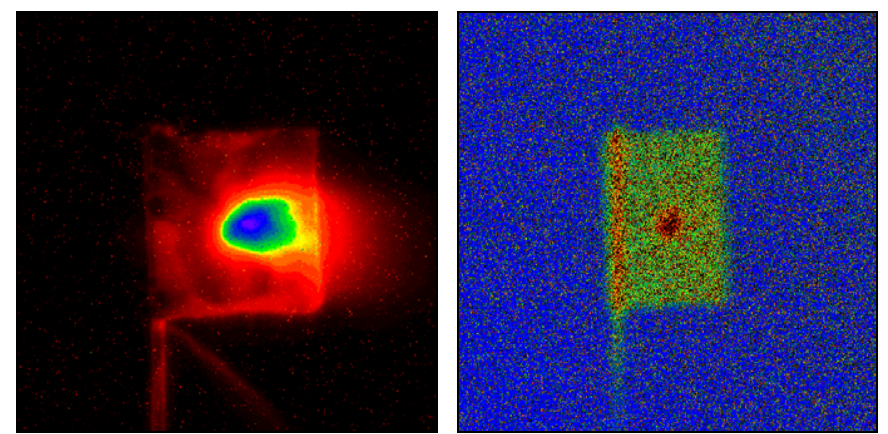

Figure 3. Image of $68 \mathrm{eV}$ XUV emission from the rear of a $330 \mu \mathrm{m}$ square, $7 \mu \mathrm{m}$ thick $\mathrm{Al} / \mathrm{Cu} / \mathrm{Al}$ target irradiated with $400 \mathrm{~J}$ laser pulse (left); corresponding $\mathrm{Cu} \mathrm{K}$-alpha emission at $8.05 \mathrm{keV}$ recorded on the same shot (right).

significant hot electron and electric/magnetic field generation and thus influence hot electron transport and $\mathrm{x}$-ray generation inside the target.

\section{Laser absorption and electron heating}

The very small interaction volume produced by a tightly focused beam inherently produces a non-1D interaction. The sharply varying radial intensity profile of the incident laser beam results in strong lateral gradients that will influence lateral thermal energy transport, both radiatively and collisionally, and lateral particle transport in the form of non-thermal or hot electrons. Figure 3 illustrates two of the diagnostics we have employed to study 2D lateral effects. The first is a time-integrated XUV imaging system consisting of a spherical mirror with a reflective multi-layer dielectric coating centred at $68 \mathrm{eV}$. The spherical mirror is used to image the rear surface of the target, via a second flat dielectric coated mirror, onto a 16-bit back-illuminated CCD camera. The second is a spherically bent crystal x-ray imager with a Bragg angle corresponding to the $\mathrm{K}$-alpha line of $\mathrm{Cu}$ at $8.05 \mathrm{keV}$ [6]. X-rays from the target at this energy are imaged onto another 16-bit back-illuminated CCD camera.

The images in figure 3 are taken from a $400 \mathrm{~J}$ pulse tightly focused on the centre of a $330 \mu \mathrm{m}$ square, $7 \mu \mathrm{m}$ thick multi-layer target ( $5 \mu \mathrm{m} \mathrm{Cu}$ coated on both sides with $1 \mu \mathrm{m} \mathrm{Al}$ ). The XUV image on the left shows the radiative emission at $68 \mathrm{eV}$ from the rear surface of the foil. To first order the intensity of emission is a function of the temperature of the foil (for a perfect blackbody the radiative intensity would be described by a Planckian thermal distribution). The foil is optically thick to $68 \mathrm{eV}$ radiation; therefore the signal in the image predominantly reflects emission from the rear surface plasma. Whilst there is a small localized intense region at the centre of the foil it is interesting to note that the rest of the foil is relatively uniformly heated. The edges of the foil are relatively sharp indicating that the emission occurs early in time before significant hydrodynamic expansion takes place. This is true except for the plume extending to the right of the target. This plume may be from the front side plasma extending beyond the target or from late-time expansion of the central hotspot at the rear surface.

The right-hand side image in figure 3 shows the corresponding data from the $8.05 \mathrm{keV}$ $\mathrm{x}$-ray imager. This image shows the $2 \mathrm{D}$ spatial distribution of the $\mathrm{Cu} \mathrm{K}$-alpha $\mathrm{x}$-ray emission. The diagnostic views the target in the horizontal plane, but at an angle of $54^{\circ}$ to the target normal, thus explaining the foreshortening of the foil in the horizontal direction. The target 
is optically thin to these $\mathrm{x}$-rays so they are able to escape from the entire $5 \mu \mathrm{m}$ thickness of $\mathrm{Cu}$. Again we observe a small hotspot within an otherwise very uniformly emitting foil. This would indicate that the hot electrons generating the $\mathrm{K}$-alpha $\mathrm{X}$-rays may rapidly settle to a uniform spatial distribution over a foil of this size (aside from the central intense spot). X-ray spectral measurements were also obtained on these shots in the $8.0-8.2 \mathrm{keV}$ region using a time-integrated x-ray crystal (HOPG) spectrometer. In these spectra x-rays are observed at shorter wavelengths than the cold $\mathrm{K}$-alpha line. The $\mathrm{x}$-rays correspond to transitions made in multiply-ionised $\mathrm{Cu}$ atoms where the stripping of outer valence electrons leads to a small increase in the energy gap between the $n=1$ and $n=2$ levels. The resulting shift in wavelength of the $\mathrm{K}$-alpha line is thus correlated to the ionisation state of the material and can be used as a diagnostic of the mean ionisation and temperature. These data are reported in Gregori et al [7], wherein the authors infer temperatures on the order of $200 \mathrm{eV}$ reached in the solid density tamped Copper.

\section{Proton beam generation}

In a thin foil target the relativistic electrons driven into the target emerge at the rear surface and through charge separation create an extremely high strength electric field normal to the target surface. This field is capable of ionizing atoms at the rear surface and accelerating them to a significant fraction of the speed of light, of the order of a picosecond in time. The presence of hydrogen on the target surface, in the form of hydrocarbons, results in the preferential acceleration of protons (bare hydrogen nuclei) as the lightest ion present. The proton beams have some remarkable properties, including laser-to-proton energy conversion efficiencies of several per cent, peak proton energies exceeding $50 \mathrm{MeV}$ and a high degree of directionality with very low transverse emittance $[8,9,10]$.

In this experiment the proton beam was characterized using a multi-layer stack of RCF placed behind the target along the axis defined by the target normal. Each layer of RCF measures the radiation dose or energy deposited within it by the beam of protons. Protons with higher energy travel through more layers before losing all their energy; thus, by measuring the deposited energy in each successive RCF layer in the stack one can reconstruct the energy spectrum of the proton beam.

Figure 4 shows the proton energy spectrum recorded from a $20 \mu \mathrm{m}$ thick $\mathrm{Cu}$ foil irradiated with a $400 \mathrm{~J}$ pulse. For this shot the laser spot was defocused by moving the target $200 \mu \mathrm{m}$ out of the plane of best focus. Whilst reducing the intensity on the target it has been found to produce a more spatially uniform proton beam. The solid line in figure 4 is a best fit using an exponential energy distribution with a temperature $k T=3.0 \mathrm{MeV}$ and a total proton number of 7E13. The lowest energy data point is at $7.8 \mathrm{MeV}$. At lower energies the energy spectrum may not, of course, necessarily follow this fit and thus the total proton number is uncertain. Above $7.8 \mathrm{MeV}$ however one can infer from the plot a total of $8.6 \mathrm{~J}$ of energy in the proton beam, equivalent to a laser-to-proton conversion efficiency of just over $2 \%$.

In addition to the energy spectrum we also characterized the spatial characteristics of the beam at the source. This was done by using a $20 \mu \mathrm{m}$ thick $\mathrm{Cu}$ foil pre-fabricated with a linear groove structure machined on the rear surface. The grooves have a separation of $3 \mu \mathrm{m}$ and their pattern becomes superimposed on the spatial profile of the proton beam during the rapid initial acceleration period. This technique has been used previously to successfully characterize aspects of the spatial distribution of the electrons and protons during the acceleration process [11].

Figure 5 shows a layer of RCF corresponding to a mean proton energy of $15 \mathrm{MeV}$. The linear pattern of the grooves, oriented at $45^{\circ}$, can clearly be seen in the spatial profile of 


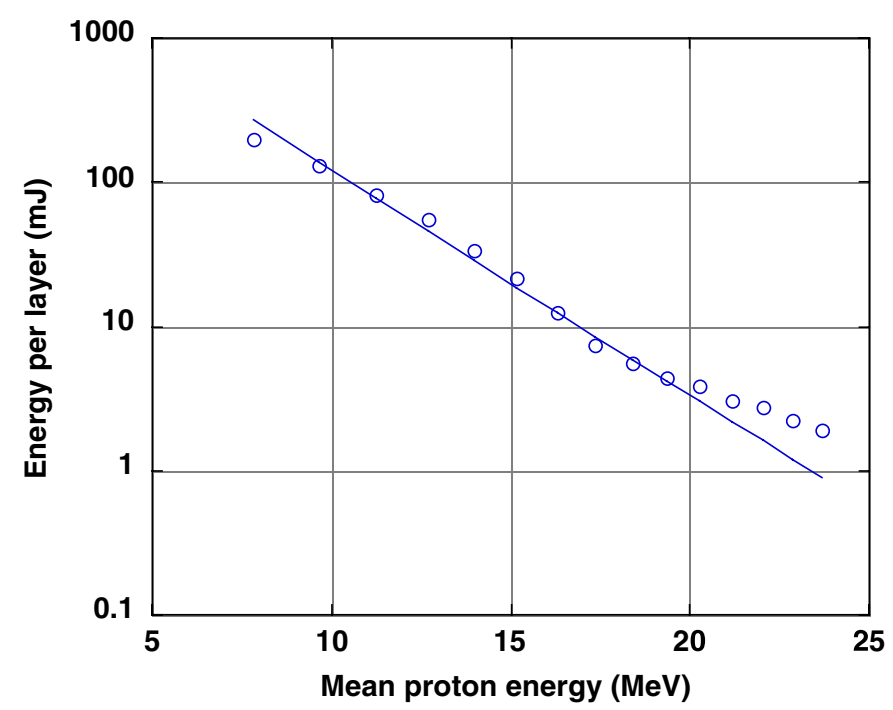

Figure 4. Proton energy spectrum recorded on $\mathrm{RCF}$ for a $400 \mathrm{~J}$ pulse incident on a $20 \mu \mathrm{m}$ thick $\mathrm{Cu}$ foil (O). The solid line is a fit to the data calculated using 7E13 protons in an exponential energy distribution with $k T=3.0 \mathrm{MeV}$.

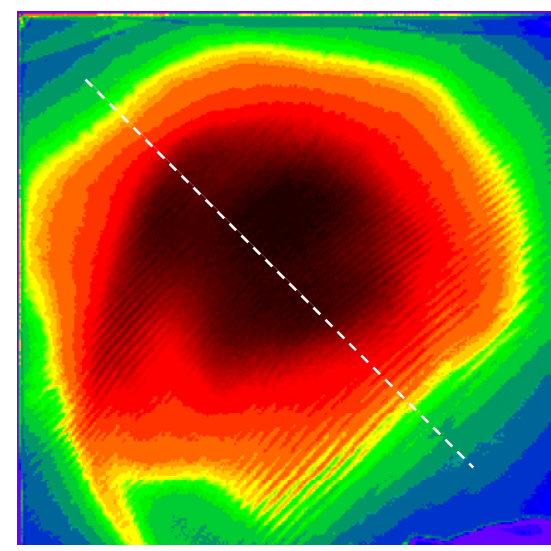

Figure 5. Image of proton beam from 10th layer of RCF, corresponding to a mean proton energy of $15 \mathrm{MeV}$. The $45^{\circ}$ linear pattern seen in the beam corresponds to the groove pattern machined into the rear surface of the target foil.

the proton beam. The outer edge of the proton beam spans 117 grooves. With a $3 \mu \mathrm{m}$ groove separation this implies that the protons originate from a source size of $350 \mu \mathrm{m}$ diameter at the target surface. The FWHM of the beam spans 69 grooves, corresponding to a $206 \mu \mathrm{m}$ diameter. The source size is actually a function of the proton energy; thus the source emitting area will be larger for proton energies below $15 \mathrm{MeV}$, and smaller for higher energies.

\section{Proton heating}

Recent experiments have demonstrated that laser-generated proton beams are sufficiently intense so as to heat solid foils to tens of electronvolts temperatures on impact [12,13]. Proton heating provides a new method for very rapid heating of solid density materials (picosecond 

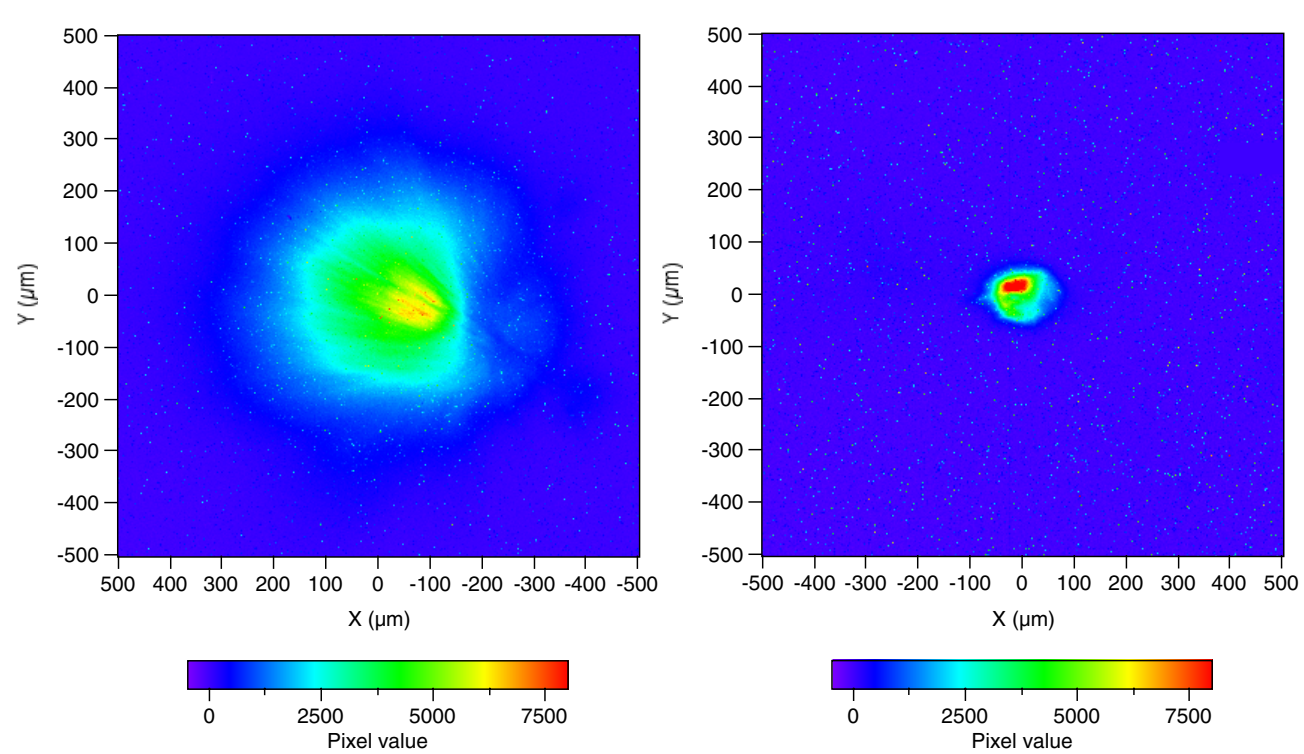

Figure 6. $68 \mathrm{eV}$ XUV emission from the rear of proton-heated foils in $(a)$ planar and $(b)$ focusing geometries. Data are plotted on the same colour scale to facilitate direct comparison of observed emission intensity.

timescales). Since multi-mega-electron-volt protons have ranges of tens to hundreds of microns they deposit their energy fairly uniformly over that distance (until near the end of the range where the rate of energy deposition increases in the form of a Bragg peak). These properties suggest that proton heating could prove to be a useful tool for creating relatively uniform high energy density plasmas for quantitative study.

We investigated two geometries for proton heating in this experiment: planar and focusing geometries. In the planar case the proton beam was produced from a $20 \mu \mathrm{m}$ thick $\mathrm{Cu}$ foil under the same conditions described in section 5. A second $10 \mu \mathrm{m}$ thick Al foil was placed $200 \mu \mathrm{m}$ behind the first foil along the target normal. In the focusing geometry the $20 \mu \mathrm{m}$ thick $\mathrm{Cu}$ foil was shaped in the form of a hemisphere with a $1 \mathrm{~mm}$ diameter. A second foil (a multilayer $\mathrm{CH} / \mathrm{Al} / \mathrm{CH}$ foil of $14 \mu \mathrm{m}$ total thickness) was placed close to the focus of the hemisphere. A multi-layer foil was used in this case on the possibility of using x-rays emitted from the Al tracer layer as a diagnostic of the conditions inside the foil. The laser is incident on the outer surface of the hemisphere. Figure 6 shows the $68 \mathrm{eV}$ XUV emission imaged from the rear of the secondary foils for these two shots.

One sees that the heated region in the focusing geometry is substantially smaller, and slightly more intense (the peak counts are approximately $50 \%$ higher). The heated region in the planar case has an FWHM diameter of $280 \mu \mathrm{m}$. This corresponds closely to the measured source size of the proton beam. In the focusing case the diameter of the heated region is only $94 \mu \mathrm{m}$ FHWM indicating that the proton beam undergoes a degree of focusing or collimation. The fact that the peak counts are only $50 \%$ higher in this case despite the significant reduction in area might be explained by reduced flux in the initial proton beam generated from the curved target, or some loss of proton flux from imperfect focusing. Based on an absolute calibration of the instrument the observed signal indicates that thermal temperatures of the order of $100 \mathrm{eV}$ or more are reached. An accurate figure for the temperature however depends on detailed modelling of the hydrodynamic expansion of the plasma, taking into proper account 
time-dependence and opacity effects on the emitted radiative intensity. Such modelling is currently being performed and will provide more quantitative information on the plasma conditions reached in the proton-heated foils.

\section{Conclusions}

In this paper we give a brief overview of a large collaborative experimental campaign carried out on the Rutherford Appleton Laboratory 500 J Petawatt laser. An extensive suite of diagnostics was employed to enable as comprehensive a set of measurements as possible of the X-ray, XUV, optical, electron, proton, and neutron emission from the plasma created by the irradiation of a solid target by a high intensity sub-picosecond laser pulse. This method of experimentally characterising the plasma through multiple and varied channels on a single shot may provide a useful means for benchmarking the increasingly sophisticated laser-plasma interaction codes being developed for the relativistic regime.

\section{Acknowledgments}

The authors wish to thank the staff of the Central Laser Facility, CCLRC Rutherford Appleton Laboratory. This work was performed under the auspices of the US Department of Energy by the University of California, Lawrence Livermore National Laboratory, under contract W-7405-ENG-48.

\section{References}

[1] Perry M D et al 1999 Opt. Lett. 24160

[2] Hatchett S P et al 2000 Phys. Plasmas 72076

[3] Krushelnik K et al 2000 Phys. Plasmas 72055

[4] Norreys P A et al 2004 Plasma Phys. Control. Fusion 46 B13

[5] Danson C et al IAEA J. Nucl. Fusion at press

[6] Koch J A et al 2003 Rev. Sci. Instrum. 742130

[7] Gregori G et al 2005 Contrib. Plasma Phys. 45 284-92

[8] Clark E L et al 2000 Phys. Rev. Lett. 84670

[9] Snavely R A et al 2000 Phys. Rev. Lett. 852945

[10] Mackinnon A J et al Phys. Rev. Lett. 88215006

[11] Cowan T E et al 2004 Phys. Rev. Lett. 92204801

[12] Patel P K et al 2003 Phys. Rev. Lett. 92125004

[13] Snavely R A et al 2003 Proc. 3rd Int. IFSA Conf. (Monterey, CA) 